\title{
Erratum to: Fertility-sparing for young patients with gynecologic cancer: How MRI can guide patient selection prior to conservative management
}

\author{
Sinead H. McEvoy, ${ }^{1,6}$ Stephanie Nougaret, ${ }^{1,7,8}$ Nadeem R. Abu-Rustum, ${ }^{2}$ \\ Hebert Alberto Vargas, ${ }^{1}$ Elizabeth A. Sadowski, ${ }^{3}$ Christine O. Menias, ${ }^{4}$ Fuki Shitano, ${ }^{1,9}$ \\ Shinya Fujii, ${ }^{5}$ Ramon E. Sosa, ${ }^{1}$ Joanna G. Escalon, ${ }^{1,10}$ Evis Sala, ${ }^{1}$ Yulia Lakhman ${ }^{1}$ \\ ${ }^{1}$ Present address: Department of Radiology, Memorial Sloan Kettering Cancer Center, New York, NY, USA \\ ${ }^{2}$ Gynecologic Service, Department of Surgery, Memorial Sloan Kettering Cancer Center, New York, NY, USA \\ ${ }^{3}$ Department of Radiology, University of Wisconsin, Madison, WI, USA \\ ${ }^{4}$ Department of Diagnostic Radiology, Mayo Clinic, Phoenix, AZ, USA \\ ${ }^{5}$ Division of Radiology, Department of Pathophysiological and Therapeutic Science, Faculty of Medicine, Tottori University, \\ Yonago, Japan \\ ${ }^{6}$ Present address: Department of Radiology, The Christie NHS Foundation, 550 Wilmslow Rd, Manchester, M20 4BX, UK \\ ${ }^{7}$ Present address: Department of Radiology, Institut Régional du Cancer de Montpellier, Montpellier, France \\ ${ }^{8}$ IRCM, Institut de Recherche en Cancérologie de Montpellier, INSERM, U1194, Montpellier, France \\ ${ }^{9}$ Present address: Department of Diagnostic Imaging and Nuclear Medicine, Graduate School of Medicine, Kyoto University, \\ Kyoto, Japan \\ ${ }^{10}$ Present address: Department of Radiology, New York-Presbyterian Hospital-Weill Cornell Medical Center, New York, NY, USA
}

\section{Erratum to: Abdom Radiol (2017) DOl: 10.1007/s00261-017-1179-3}

The original version of this article unfortunately contained mistakes. The Figs. 7D, 7E and 7F were missing in the article and arrows were missing in the Figs. 6C, 8B and $11 \mathrm{C}$. The year of publication and volume number for references 19, 79 and 87 have been updated. Also, the
Table 2 layout has been improved for better readability. The Publisher apologizes for the mistakes and the inconvenience caused.

The corrected Figs. 6-8 and 11 are provided here.

The original article was corrected.

The online version of the original article can be found under doi: 10.1007/s00261-017-1179-3. 
Table 2. Tailored MR Imaging Protocols for the Initial Work-Up of Gynecologic Cancers and Adnexal Lesions

Patient preparation, positioning, and coil selection

$\checkmark$ Fast 4-6 h before

Empty the bladder

$\checkmark$ Anti-peristaltic agent administration (IM or IV)

$\checkmark$ Supine position

$\checkmark \geq 1.5$-Tesla magnet

$\checkmark$ Multi-channel phased-array receiver coil

$\checkmark$ Optional

- Endovaginal receiver coils for small cervical lesions

- Vaginal opacification with gel for cervical cancer

Tailored MR imaging protocols

Large FOV T1WI \pm T2WI

$\checkmark$ T1WI without and with fat saturation
- From the top of the kidneys to the iliac crests (Upper abdomen)

- Pelvis, Axial plane

\begin{tabular}{|c|c|c|c|}
\hline & Cervical cancer & Endometrial cancer & Adnexal mass \\
\hline $\boldsymbol{V}$ High-resolution small FOV T2WI & $\begin{array}{l}\text { - Sagittal plane } \\
\text { - Axial oblique plane (perpendicular to } \\
\text { the endocervical canal) } \\
\text { - Optional: Coronal oblique plane (par- } \\
\text { allel to the endocervical canal) }\end{array}$ & $\begin{array}{l}\text { - Sagittal plane } \\
\text { - Axial oblique plane (perpen- } \\
\text { dicular to the endometrial } \\
\text { cavity) }\end{array}$ & $\begin{array}{l}\text { - Sagittal plane } \\
\text { - Axial plane } \\
\text { - Axial oblique plane } \\
\quad \text { (ovarian axis) }\end{array}$ \\
\hline $\boldsymbol{V}$ DWI and ADC map & $\begin{array}{l}\text { - Sagittal plane } \\
\text { - Axial oblique plane in the same orien- } \\
\text { tations as axial oblique T2WI } \\
\text { - Optional: Coronal oblique DWI (par- } \\
\text { allel to the endocervical canal) }\end{array}$ & $\begin{array}{l}\text { - Sagittal plane } \\
\text { - Axial oblique plane in the } \\
\text { same orientations as axial } \\
\text { oblique T2WI }\end{array}$ & - Axial plane \\
\hline $\boldsymbol{v}$ DCE-MRI & $\begin{array}{l}\text { - Useful for assessment of small tumors } \\
\text { - DCE-MRI: Sagittal plane } \\
\text { - Delayed CE: Axial oblique plane }\end{array}$ & $\begin{array}{l}\text { - DCE-MRI: Sagittal plane } \\
\text { - Delayed CE: Axial oblique } \\
\text { plane }\end{array}$ & - Axial plane \\
\hline
\end{tabular}

$I M$ intramuscular, $I V$ intravenous, $M R$ magnetic resonance, $F O V$ field of view, $T 1 W I$ T1-weighted imaging, $T 2 W I$ T2-weighted imaging, $D W I$ diffusion weighted imaging, $A D C$ apparent diffusion coefficient, $D C E-M R I$ dynamic contrast-enhanced MRI, $C E$ contrast enhancement 


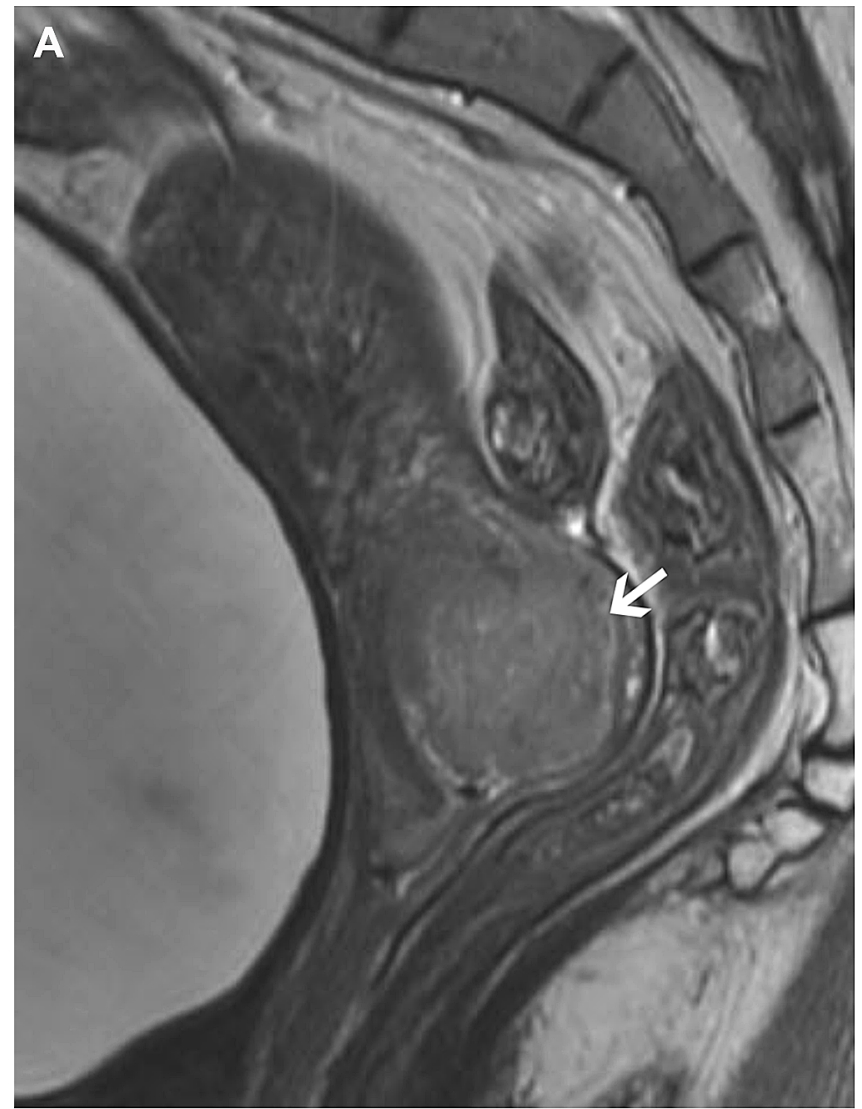

Fig. 6. 37-year-old woman with Stage IB1 cervical cancer. A Sagittal T2WI demonstrates $2.7-\mathrm{cm}$ endophytic intermediate-SI tumor (white arrow). B, C. Axial oblique T2WI and fused $(\mathrm{T} 2 \mathrm{WI}+\mathrm{DWI})$ image, respectively, show tumor exten-
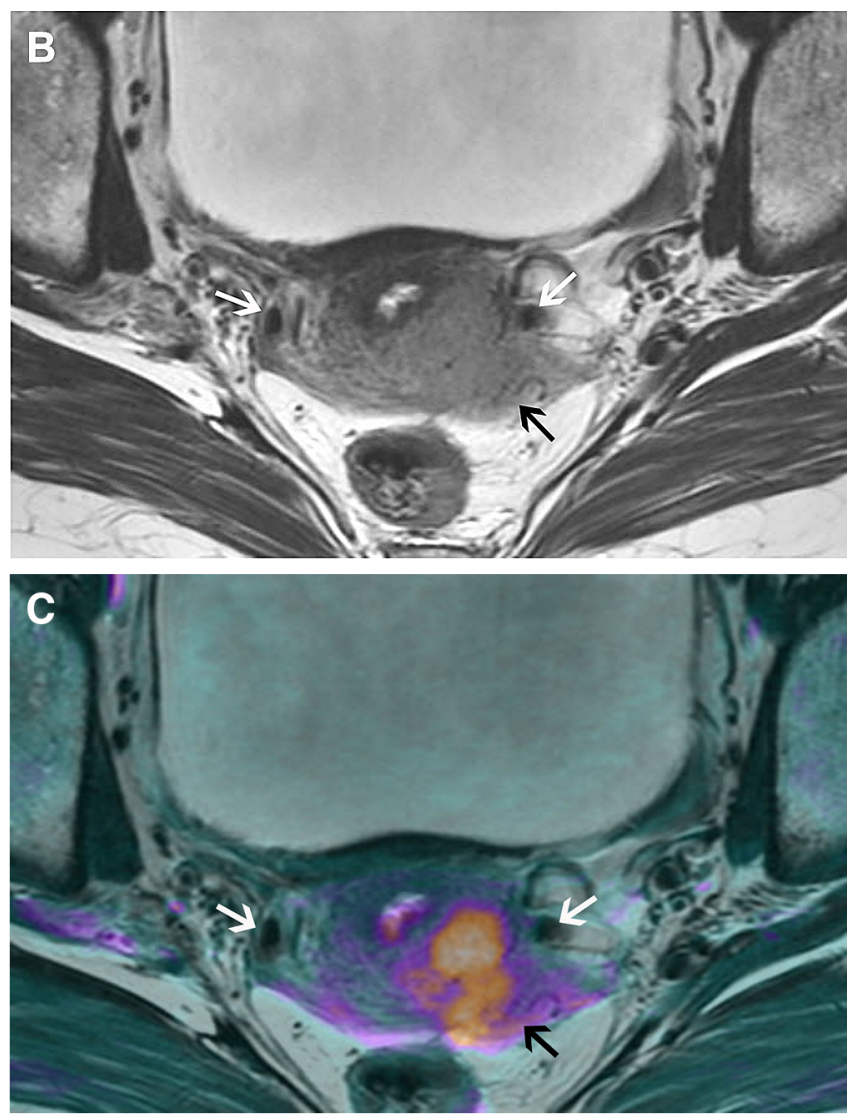

sion to the internal os and left parametrial invasion (black arrows). The internal os is demarcated as the entrance of the uterine vessels (white arrows). 

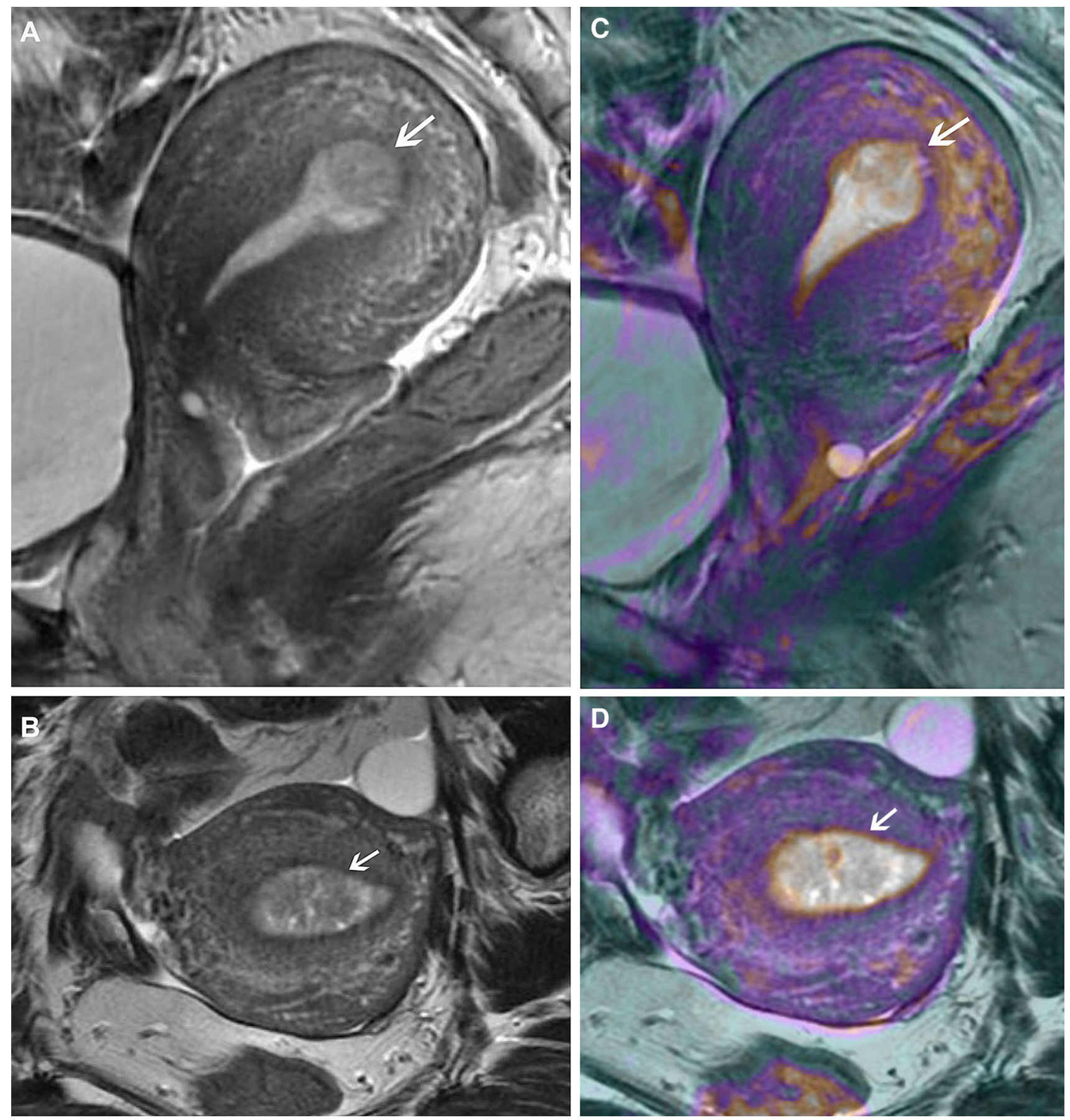

Fig. 7. 40-year-old woman with new onset menorrhagia and D\&C demonstrating FIGO grade 1 endometrioid adenocarcinoma. A, B. Sagittal and axial oblique T2WI demonstrate intermediate-SI tumor in the endometrial cavity and preserved smooth interface between the tumor and low-SI junctional zone (white arrows). C, D. Sagittal and axial oblique fused
(T2WI + DWI) images confirm smooth interface between the tumor and junctional zone (white arrows). E, F. Sagittal early (E) and delayed DCE-MRI (F) images show intact band of junctional zone enhancement (black arrows) and smooth tumor-to-myometrium interface (black arrows), respectively. 

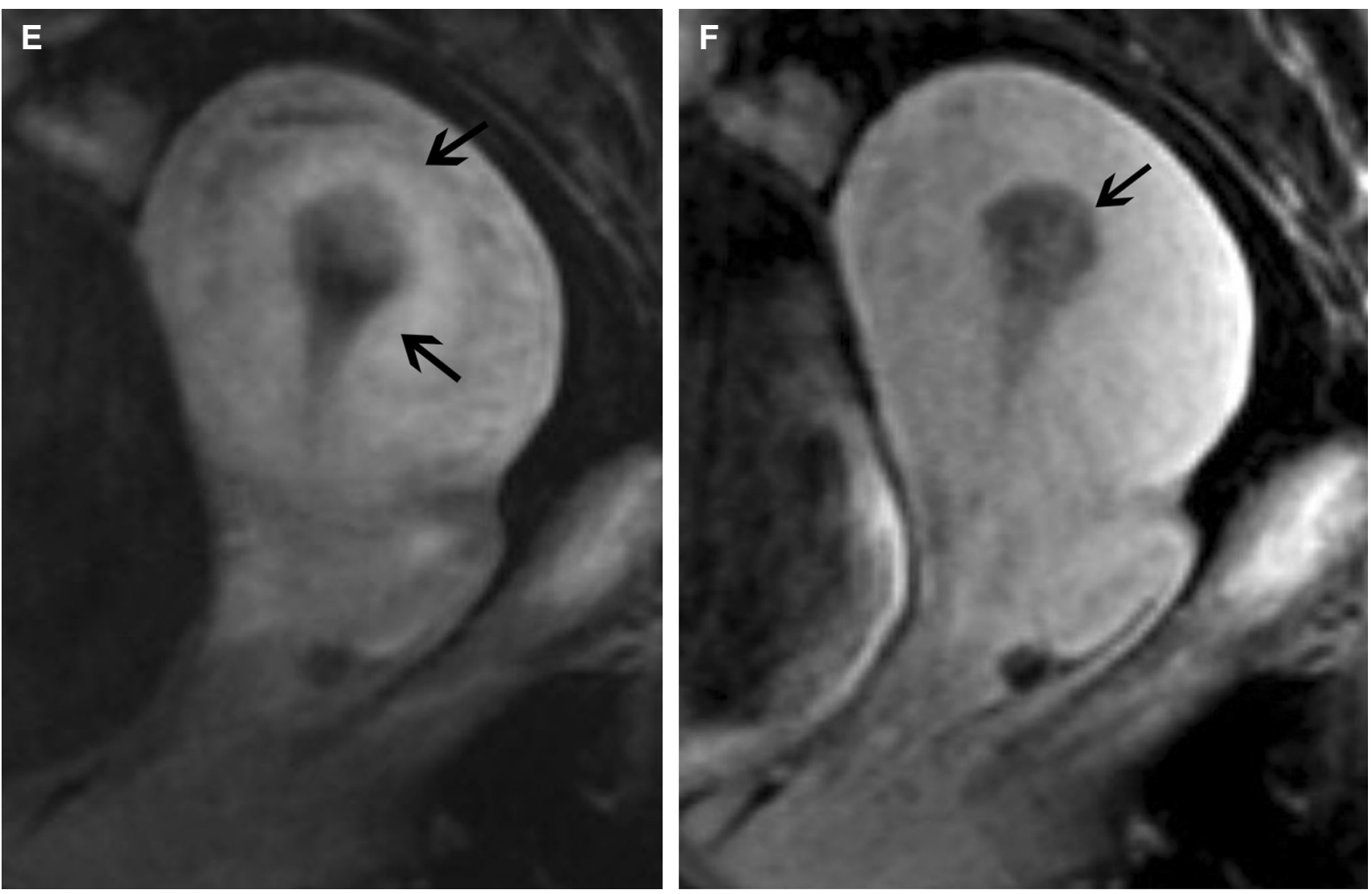

Fig. 7. continued. 

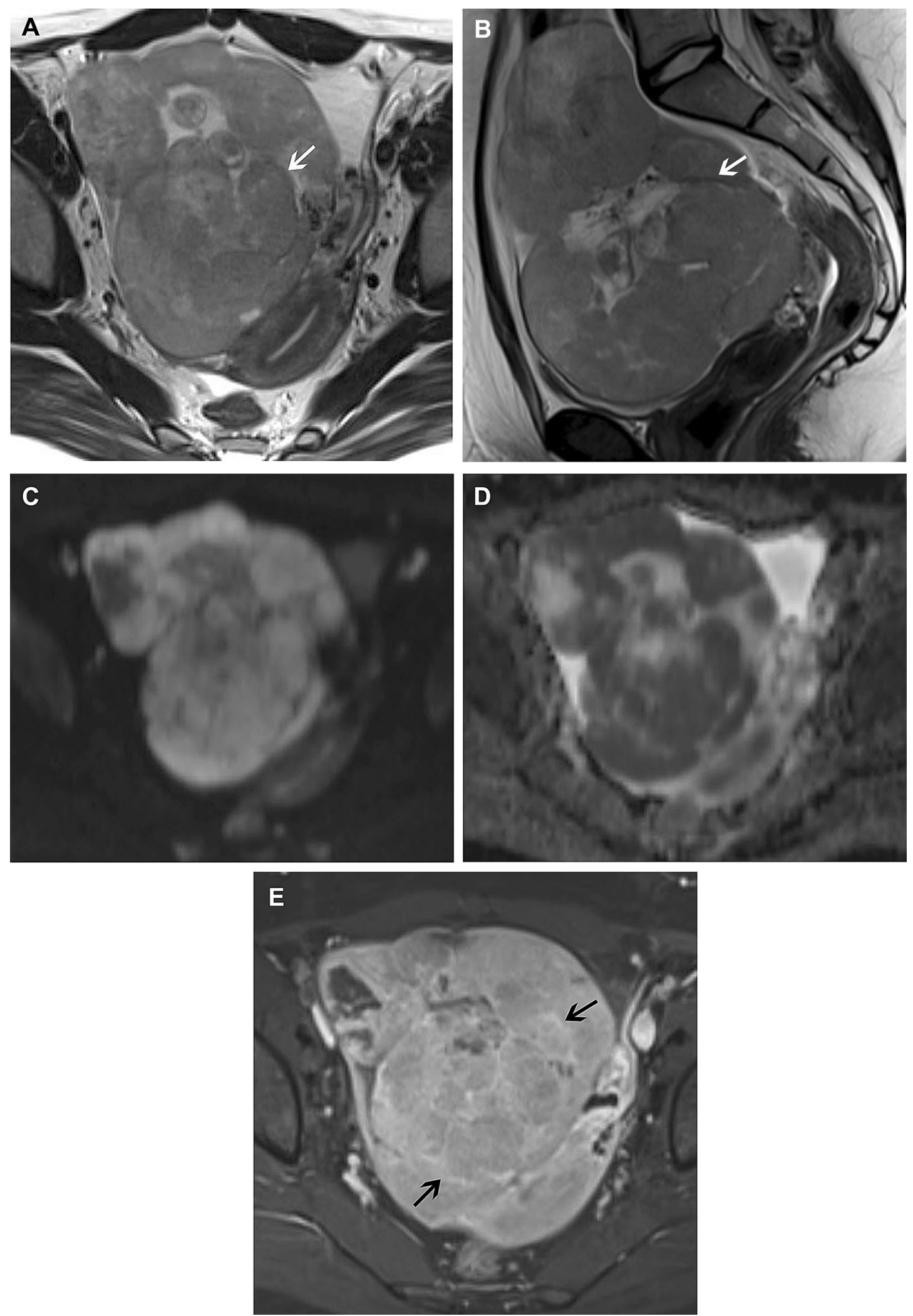

Fig. 8. 23-year-old woman with dysgerminoma who presented with low back pain and elevated serum LDH and $\beta$ HCG. A, B Axial and sagittal T2WI show solid intermediate-SI adnexal mass with low-SI fibrovascular septa (white arrows).
C, D Axial DWI and ADC map demonstrate diffusion restriction. E Axial contrast-enhanced image shows solid enhancing adnexal mass with avidly enhancing fibrovascular septa (black arrows). 

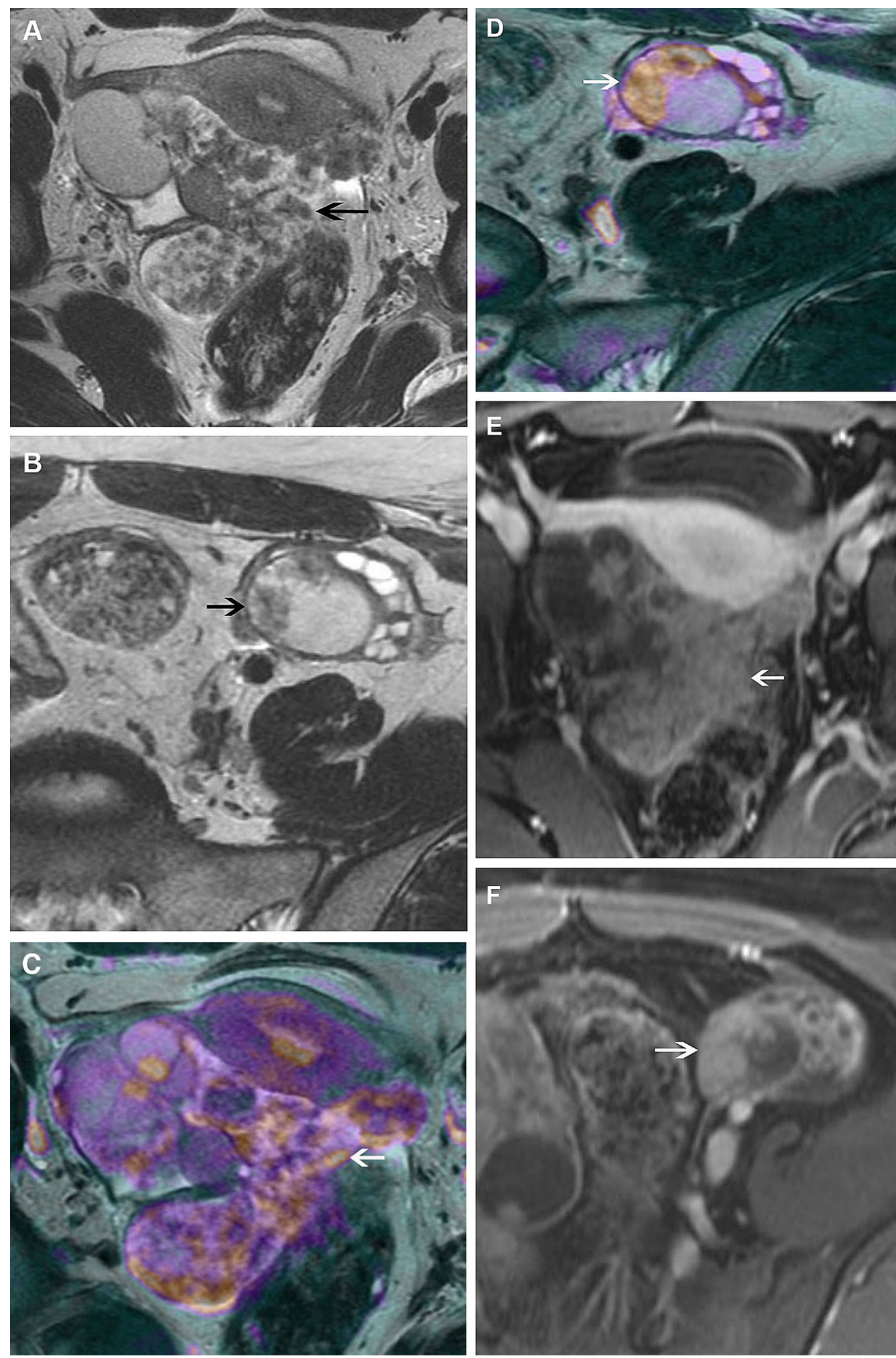

Fig. 11. 20-year-old woman with bilateral serous borderline

small cystic lesion with small intermediate-SI papillary proovarian tumors who presented with lower abdominal pain and elevated serum CA-125 level. A Axial T2WI of the right adnexa shows cystic and solid right adnexal mass with large branching papillary projections (black arrow) extending into the pouch of Douglas. B Axial T2WI of the left adnexa shows jections (black arrow). C, D Axial fused (T2WI + DWI) images demonstrate restricted diffusion within papillary projections (white arrows). E, F Axial contrast-enhanced images show enhancement within these papillary projections (white arrows). 


\section{Reference}

19. Milgrom SA, Vargas HA, Sala E, et al. (2013) Acute effects of pelvic irradiation on the adult uterus revealed by dynamic contrast-enhanced MRI. Br J Radiol 86(1031):20130334

79. Shen G, Zhou H, Jia Z, Deng H (2015) Diagnostic performance of diffusion-weighted MRI for detec- tion of pelvic metastatic lymph nodes in patients with cervical cancer: a systematic review and metaanalysis. Br J Radiol 88(1052):20150063

87. Morice P, Leary A, Creutzberg C, Abu-Rustum N, Darai E (2016)Endometrial cancer. Lancet 387(10023):1094-1108 